\title{
EXPLICIT MULTIVARIABLE MEROMORPHIC SERIES
}

\author{
SHREERAM S. ABHYANKAR, NICHOLAS J. FIREMAN, AND NATHAN C. MOSES \\ (Communicated by Irena Peeva)
}

ABSTRACT. In this paper, we present a constructive approach to multivariable meromorphic functions.

\section{INTRODUCTION}

For a field $K$, let $Y_{1}, \ldots, Y_{m}$ be indeterminates over $K$ where $m \in \mathbb{N}_{+}=$the set of all positive integers. Then for the polynomial and power series rings we have

$$
K\left[Y_{1}, \ldots, Y_{m}\right] \subset K\left[\left[Y_{1}, \ldots, Y_{m}\right]\right]
$$

and for their quotient fields we have

$$
K\left[Y_{1}, \ldots, Y_{m}\right] \subset K\left(Y_{1}, \ldots, Y_{m}\right)=\text { the rational function field }
$$

and

$$
K\left[\left[Y_{1}, \ldots, Y_{m}\right]\right] \subset K\left(\left(Y_{1}, \ldots, Y_{m}\right)\right)=\text { the meromorphic function field. }
$$

Hence we get the subfield inclusion

$$
K\left(Y_{1}, \ldots, Y_{m}\right) \subset K\left(\left(Y_{1}, \ldots, Y_{m}\right)\right) .
$$

In case of $m=1$, members of $K\left(\left(Y_{1}\right)\right)$ have concrete representations as meromorphic series, i.e., expressions of the form

$$
g\left(Y_{1}\right)=\sum_{i \in \mathbb{Z}} b_{i} Y_{1}^{i} \text { with } b_{i} \in K
$$

such that the support $\operatorname{Supp}(g)=\left\{i \in \mathbb{Z}: b_{i} \neq 0\right\}$ has only a finite number of negative integers; see page 69 of [Ab1] or page 39 of Ab2]. We may ask the explicitization question: is there such an explicit representation of members of $K\left(\left(Y_{1}, \ldots, Y_{m}\right)\right)$ for $m>1$ ? To move towards an answer, consider the set $K\left\langle Y_{1}, \ldots, Y_{m}\right\rangle$ consisting of all expressions of the form

$$
f\left(Y_{1}, \ldots, Y_{m}\right)=\sum_{\left(i_{1}, \ldots, i_{m}\right) \in \mathbb{Z}^{m}} a_{i_{1} \ldots i_{m}} Y_{1}^{i_{1}} \ldots Y_{m}^{i_{m}} \text { with } a_{i_{1} \ldots i_{m}} \in K
$$

In an obvious manner $K\left\langle Y_{1}, \ldots, Y_{m}\right\rangle$ can be identified with $K^{\mathbb{Z}^{m}}=$ the set of all maps $\mathbb{Z}^{m} \rightarrow K$, and we define the support of $f$ by putting

$$
\operatorname{Supp}(f)=\left\{\left(i_{1}, \ldots, i_{m}\right) \in \mathbb{Z}^{m}: a_{i_{1} \ldots i_{m}} \neq 0\right\} .
$$

In a natural manner we convert $K\left\langle Y_{1}, \ldots, Y_{m}\right\rangle$ into a $K$-vector-space; in view of the one complex variable theorem on laurent expansion of functions in a punctured

Received by the editors May 16, 2012.

2010 Mathematics Subject Classification. Primary 14A05.

Key words and phrases. Multivariable, meromorphic function, meromorphic series. 
disc, we call this vector space the laurent vector space (in $Y_{1}, \ldots, Y_{m}$ over $K$ ). Identifying $K\left[\left[Y_{1}, \ldots, Y_{m}\right]\right]$ with $K^{\mathbb{N}^{m}}$, where $\mathbb{N}=$ the set of all nonnegative integers, we have a natural monomorphism of $K$-vector-spaces

$$
\phi: K\left[\left[Y_{1}, \ldots, Y_{m}\right]\right] \rightarrow K\left\langle Y_{1}, \ldots, Y_{m}\right\rangle
$$

with

$$
\operatorname{im}(\phi)=\left\{f \in K\left\langle Y_{1}, \ldots, Y_{m}\right\rangle: \operatorname{Supp}(f) \in \mathbb{N}^{m}\right\} .
$$

Remark 1.1. Let $\operatorname{lex}\left(\mathbb{Z}^{m}\right)$ denote the ordered abelian group obtained by lexicographically ordering $\mathbb{Z}^{m}$ whereby: $\left(i_{1}, \ldots, i_{m}\right)<\left(j_{1}, \ldots, j_{m}\right)$ means for some $p \in$ $\{1, \ldots, m\}$ we have $i_{1}=j_{1}, \ldots, i_{p-1}=j_{p-1}, i_{p}<j_{p}$. We put

$$
K\left(\left\langle Y_{1}, \ldots, Y_{m}\right\rangle\right)=\left\{f \in K\left\langle Y_{1}, \ldots, Y_{m}\right\rangle: \operatorname{Supp}(f) \text { is well ordered }\right\}
$$

and we call $K\left(\left\langle Y_{1}, \ldots, Y_{m}\right\rangle\right)$ the lexomorphic field (in $Y_{1}, \ldots, Y_{m}$ over $K$ ). Now

$$
\operatorname{im}(\phi)=\phi\left(K\left[\left[Y_{1}, \ldots, Y_{m}\right]\right]\right) \subset K\left(\left\langle Y_{1}, \ldots, Y_{m}\right\rangle\right)
$$

because, in the lexicographic ordering, $\mathbb{N}^{m}$ is a well ordered subset of $\mathbb{Z}^{m}$. To see this let $T$ be any nonempty subset of $\mathbb{N}^{m}$. Let

$$
\text { the projection } \pi_{j}: \mathbb{Z}^{m} \rightarrow \mathbb{Z} \text { be given by } i=\left(i_{1}, \ldots, i_{m}\right) \mapsto i_{j} \text {. }
$$

Using the fact that $\mathbb{N}$ is well ordered we can inductively define

$$
\left\{\begin{array}{l}
t_{1}=\min \left\{\pi_{1}(i): i \in T\right\}, \ldots, t_{j}=\min \left\{\pi_{j}(i): i \in T \cap \bigcap_{1 \leq l \leq j-1} \pi_{l}^{-1}\left(t_{l}\right)\right\}, \\
\ldots, t_{m}=\min \left\{\pi_{m}(i): i \in T \cap \bigcap_{1 \leq l \leq m-1} \pi_{l}^{-1}\left(t_{l}\right)\right\}
\end{array}\right.
$$

and then clearly $\left(t_{1}, \ldots, t_{m}\right)=\min (T)$.

Remark 1.2. In Section 2 we shall answer the explicitization question by showing that: $K\left(\left\langle Y_{1}, \ldots, Y_{m}\right\rangle\right)$ can be made into an overfield of $K$ and $\phi$ can be uniquely extended to a ring monomorphism

$$
\Phi: K\left(\left(Y_{1}, \ldots, Y_{m}\right)\right) \rightarrow K\left(\left\langle Y_{1}, \ldots, Y_{m}\right\rangle\right)
$$

thus expanding all the members of $K\left(\left(Y_{1}, \ldots, Y_{m}\right)\right)$ as multivariable meromorphic series.

Remark 1.3. In Section 3 we shall indicate how, by using Galois theory, it can be shown that, if $m>1$, then the field $K\left(\left\langle Y_{1}, \ldots, Y_{m}\right\rangle\right)$ is much larger than the subfield $\Phi\left(K\left(\left(Y_{1}, \ldots, Y_{m}\right)\right)\right)$; the details of the Galois theory proof will be given in another paper. In Section 3 we shall also describe some other aspects of the extensions

$$
\Phi\left(K\left(\left(Y_{1}, \ldots, Y_{m}\right)\right)\right) \subset K\left(\left\langle Y_{1}, \ldots, Y_{m}\right\rangle\right) \subset K\left\langle Y_{1}, \ldots, Y_{m}\right\rangle .
$$

In Section 5 , by a combinatorial argument we shall show that if $m>1$, then $K\left(\left\langle Y_{1}, \ldots, Y_{m}\right\rangle\right)$ is an infinite dimensional vector space over $\Phi\left(K\left(\left(Y_{1}, \ldots, Y_{m}\right)\right)\right)$. In Section 6, by extending this argument we shall show that if $m>1$, then $K\left(\left\langle Y_{1}, \ldots, Y_{m}\right\rangle\right)$ has infinite transcendence degree over $\Phi\left(K\left(\left(Y_{1}, \ldots, Y_{m}\right)\right)\right)$.

Remark 1.4. To sketch a proof of the first half of Remark 1.2, given any ordered abelian group $G$, as on page 41 of [Ab2], for all $A \in K^{G}$ we define

$$
\operatorname{Supp}(A)=\{g \in G: A(g) \neq 0\}
$$

and we put

$$
K((X))_{G}=\left\{A \in K^{G}: \operatorname{Supp}(A) \text { is well ordered }\right\} .
$$


We call $K((X))_{G}$ the unomorphic field (in $X$ over $K$ relative to $G$ ) and, moreover, for all $A \in K((X))_{G}$ we define

$$
\operatorname{ord}(A)= \begin{cases}\min \operatorname{Supp}(A) & \text { if } A \neq 0 \\ \infty & \text { if } A=0\end{cases}
$$

We put

$$
K[[X]]_{G}=\left\{A \in K((X))_{G}: \operatorname{ord}(A) \geq 0\right\}
$$

and we call $K[[X]]_{G}$ the unomorphic $\operatorname{ring}$ (in $X$ over $K$ relative to $G$ ). On page 42 of [Ab2] (with more details on pages 674-677 of [Ab2]) it is proved that $K((X))_{G}$ is indeed a field and $A \mapsto \operatorname{ord}(A)$ gives a valuation $K \rightarrow G \cup\{\infty\}$ whose valuation ring is $K[[X]]_{G}$. Generalizing what we have said on page 42 of [Ab2], concretely speaking, a typical member of $K^{G}$ can be written as

$$
A(X)=\sum_{i \in G} A_{i} X^{i}
$$

where we are writing $A_{i}$ for the previous $A(i)$. The $K$-vector-space $K^{G}$ may be denoted by $K\langle X\rangle_{G}$ and called the laurent vector space (in $X$ over $K$ relative to $G)$. Clearly

$$
f \mapsto A(X)=\sum_{i=\left(i_{1}, \ldots, i_{m}\right) \in \mathbb{Z}^{m}} A_{i} X^{i} \text { with } A_{i}=a_{i_{1}, \ldots, i_{m}}
$$

gives a $K$-vector-space bijection

$$
\theta: K\left\langle Y_{1}, \ldots, Y_{m}\right\rangle \rightarrow K\langle X\rangle_{\operatorname{lex}\left(\mathbb{Z}^{m}\right)}
$$

and remembering that $K\left(\left\langle Y_{1}, \ldots, Y_{m}\right\rangle\right) \subset K\left\langle Y_{1}, \ldots, Y_{m}\right\rangle$ we have

$$
\theta\left(K\left(\left\langle Y_{1}, \ldots, Y_{m}\right\rangle\right)\right)=K((X))_{\operatorname{lex}\left(\mathbb{Z}^{m}\right)} .
$$

This takes care of the first part of Remark 1.2 saying that $K\left(\left\langle Y_{1}, \ldots, Y_{m}\right\rangle\right)$ can be made into an overfield of $K$. We put

$$
K\left[\left\langle Y_{1}, \ldots, Y_{m}\right\rangle\right]=\theta^{-1}\left(K[[X]]_{\operatorname{lex}\left(\mathbb{Z}^{m}\right)}\right)
$$

and we call this the lexomorphic ring (in $Y_{1}, \ldots, Y_{m}$ over $K$ ). In connection with Remarks 1.2 and 1.3, in Section 3 we shall show that

$$
\Phi\left(K\left[\left[Y_{1}, \ldots, Y_{m}\right]\right]\right) \subset K\left[\left\langle Y_{1}, \ldots, Y_{m}\right\rangle\right] .
$$

Remark 1.5. Here is a paradox. By a "crude" calculation based on the geometric series identity $(1-Z)\left(\sum_{n=0}^{\infty} Z^{n}\right)=1$ we surmise that

$$
\frac{1}{Y_{1}+Y_{2}}=\frac{1}{Y_{1}} \sum_{n=0}^{\infty}\left(\frac{-Y_{2}}{Y_{1}}\right)^{n}=\sum_{n=0}^{\infty}(-1)^{n} Y_{1}^{-n-1} Y_{2}^{n}
$$

and

$$
\frac{1}{Y_{1}+Y_{2}}=\frac{1}{Y_{2}} \sum_{n=0}^{\infty}\left(\frac{-Y_{1}}{Y_{2}}\right)^{n}=\sum_{n=0}^{\infty}(-1)^{n} Y_{1}^{n} Y_{2}^{-n-1} .
$$

Hence by equating the two extreme RHSs we obtain

$$
\sum_{n=0}^{\infty}(-1)^{n} Y_{1}^{-n-1} Y_{2}^{n}=\sum_{n=0}^{\infty}(-1)^{n} Y_{1}^{n} Y_{2}^{-n-1}
$$

which is absurd. 
More generally, for any positive integer $q \leq m$, a "crude" calculation based on the geometric series indicates that

$$
\frac{1}{Y_{1}+\cdots+Y_{m}}=H_{q}\left(Y_{1}, \ldots, Y_{m}\right) \in K\left\langle Y_{1}, \ldots, Y_{m}\right\rangle
$$

where

$$
H_{q}\left(Y_{1}, \ldots, Y_{m}\right)=\sum_{n=0}^{\infty}(-1)^{n} Y_{q}^{-n-1}\left(Y_{1}+\cdots+Y_{q-1}+Y_{q+1}+\cdots+Y_{m}\right)^{n} .
$$

Therefore for all $q \neq q^{\prime}$ in $\{1, \ldots, m\}$ we have $H_{q}\left(Y_{1}, \ldots, Y_{m}\right)=H_{q^{\prime}}\left(Y_{1}, \ldots, Y_{m}\right)$ which (in case of $m>1$ ) is absurd because for all $i=\left(i_{1}, \ldots, i_{m}\right) \in \operatorname{Supp}\left(H_{q}\right)$ we obviously have $i_{j} \in \mathbb{N} \Leftrightarrow j \neq q$.

In Section 4, resolution of this paradox will lead us to interesting automorphisms of $K\left\langle Y_{1}, \ldots, Y_{m}\right\rangle$ as a $K$-vector-space.

\section{EXPANSIONS OF MULTIVARIABLE MEROMORPHIC FUNCTIONS}

At the end of Remark 1.4 we have shown that $\theta\left(K\left(\left\langle Y_{1}, \ldots, Y_{m}\right\rangle\right)\right)=K((X))_{\operatorname{lex}\left(\mathbb{Z}^{m}\right)}$ and hence $K\left(\left\langle Y_{1}, \ldots, Y_{m}\right\rangle\right)$ can be made into an overfield of $K$. By Remark 1.1 we have $\phi\left(K\left[\left[Y_{1}, \ldots, Y_{m}\right]\right]\right) \subset K\left(\left\langle Y_{1}, \ldots, Y_{m}\right\rangle\right)$, and by page 42 of Ab2 multiplication in $K((X))_{\operatorname{lex}\left(\mathbb{Z}^{m}\right)}$ is defined by the "Cauchy rule". Consequently, $\phi$ induces a ring monomorphism $K\left[\left[Y_{1}, \ldots, Y_{m}\right]\right] \rightarrow K\left(\left\langle Y_{1}, \ldots, Y_{m}\right\rangle\right)$. Since $K\left(\left\langle Y_{1}, \ldots, Y_{m}\right\rangle\right)$ is field, it follows that $\phi$ can be uniquely extended to ring monomorphism

$$
\Phi: K\left(\left(Y_{1}, \ldots, Y_{m}\right)\right) \rightarrow K\left(\left\langle Y_{1}, \ldots, Y_{m}\right\rangle\right) .
$$

This completes the proof of Remark 1.2.

\section{Structure of VARIOUS RING AND FIELD EXTENSIONS}

We start by converting the lexomorphic field into the iterated meromorphic field.

Remark 3.1 (Iterated meromorphic field). Let us consider the iterated meromorphic field

$$
L_{m, 1}=K\left(\left(Y_{m}\right)\right)\left(\left(Y_{m-1)}\right) \ldots\left(\left(Y_{1}\right)\right)\right.
$$

defined, with decreasing induction, by putting

$$
L_{m, m}=K\left(\left(Y_{m}\right)\right), L_{m, m-1}=L_{m, m}\left(\left(Y_{m-1}\right)\right), \ldots, L_{m, 1}=L_{m, 2}\left(\left(Y_{1}\right)\right)
$$

where $L_{m, j}$ is temporary notation. Obviously $L_{m, j}$ may be regarded as a subset of $K\left\langle Y_{1}, \ldots, Y_{m}\right\rangle$. Let us prove that then

$$
K\left(\left\langle Y_{1}, \ldots, Y_{m}\right\rangle\right)=K\left(\left(Y_{m}\right)\right)\left(\left(Y_{m-1}\right)\right) \ldots\left(\left(Y_{1}\right)\right) .
$$

For $1 \leq j \leq m$, we may identify $K\left(\left\langle Y_{j}, \ldots, Y_{m}\right\rangle\right)$ with the set of those members of $K\left(\left\langle Y_{1}, \ldots, Y_{m}\right\rangle\right)$ whose support belongs to $P_{j}=\pi_{1}^{-1}(0) \cap \cdots \cap \pi_{j-1}^{-1}(0)$ where $P_{1}=\mathbb{Z}^{m}$; note that now the field $K\left(\left\langle Y_{j}, \ldots, Y_{m}\right\rangle\right)$ equals the intersection of $K\left\langle Y_{j}, \ldots, Y_{m}\right\rangle$ with the field $K\left(\left\langle Y_{1}, \ldots, Y_{m}\right\rangle\right)$. To prove (3.1.1), by decreasing induction of $j$ we shall show that $K\left(\left\langle Y_{j}, \ldots, Y_{m}\right\rangle\right)=L_{m, j}$ for $1 \leq j \leq m$. For $j=m$ this is obvious. Moreover, for any positive integer $j<m$, the $j$ case follows from the $j+1$ case by noting that a subset $Q$ of $P_{j}$ is well ordered iff $\pi_{j}(Q)$ is well ordered and $\pi_{j}^{-1}(R) \cap P_{j}$ is well ordered for all $R \in \pi_{j}(Q)$. 
In connection with Remark $1.4(\bullet)$ we note that, for any $f \in K\left[\left[Y_{1}, \ldots, Y_{m}\right]\right]$ we have $\operatorname{Supp}(\phi(f)) \in \mathbb{N}^{m}$ and hence $\theta(\phi(f)) \in K[[X]]_{\operatorname{lex}\left(\mathbb{Z}^{m}\right)}$. This proves Remark $1.4(\bullet)$ which we restate as

$$
\Phi\left(K\left[\left[Y_{1}, \ldots, Y_{m}\right]\right]\right) \subset K\left[\left\langle Y_{1}, \ldots, Y_{m}\right\rangle\right] .
$$

Remark 3.2 (Galois tower). For any field $L$, by $G T(L)$ we denote the set of all finite Galois groups $\operatorname{Gal}\left(L^{*}, L\right)$ of (finite) Galois extensions $L^{*}$ of $L$. We call $G T(L)$ the Galois tower of $L$. We say that $G T(L)$ is solvable (resp.: cyclic, abelian) to mean that every member of $G T(L)$ is solvable (resp.: cyclic, abelian). We say that $G T(L)$ is unsolvable to mean that some member of $G T(L)$ is unsolvable. We claim that

$$
\left\{\begin{array}{l}
\text { if } G T(K) \text { is solvable, then so is } G T\left(K\left\langle Y_{1}, \ldots, Y_{m}\right\rangle\right) \\
\text { hence, in particular, if } K \text { is either algebraically closed or finite, } \\
\text { then } G T\left(K\left\langle Y_{1}, \ldots, Y_{m}\right\rangle\right) \text { is solvable. }
\end{array}\right.
$$

Recall that $S_{m}$ is the permutation group on $m$ letters with size $\left|S_{m}\right|=m$ !. We claim that

$$
S_{m} \in G T\left(K\left(\left(Y_{1}, \ldots, Y_{m}\right)\right)\right) .
$$

We also claim that

$$
\text { if } m>1 \text {, then } G T\left(K\left(\left(Y_{1}, \ldots, Y_{m}\right)\right)\right) \text { is unsolvable. }
$$

For $m \geq 5$, clearly (3.2.2) $\Rightarrow(3.2 .3)$. Proofs of claims (3.2.1) to (3.2.3) will be given in another paper.

\section{INTERESTING VECTOR SPACE AUTOMORPHISMS}

Let $S_{m}$ be the permutation group on $(1, \ldots, m)$. For every $\sigma \in S_{m}$ we get a bijection $\widehat{\sigma}: \mathbb{Z}^{m} \rightarrow \mathbb{Z}^{m}$ which sends $i=\left(i_{1}, \ldots, i_{m}\right)$ to $\widehat{\sigma}(i)=\left(i_{\sigma(1)}, \ldots, i_{\sigma(m)}\right)$. In turn we get a bijection

$$
\widetilde{\sigma}: K^{\mathbb{Z}^{m}}=K\left\langle Y_{1}, \ldots, Y_{m}\right\rangle \rightarrow K\left\langle Y_{1}, \ldots, Y_{m}\right\rangle=K^{\mathbb{Z}^{m}}
$$

by sending every $A \in K^{\mathbb{Z}^{m}}$ to $\widetilde{\sigma}(A) \in K^{\mathbb{Z}^{m}}$ such that $\widetilde{\sigma}(A)(i)=A(\widehat{\sigma}(i))$ for all $i \in \mathbb{Z}^{m}$. Clearly $\widehat{\sigma}$ is a $K$-linear automorphism of $K\left\langle Y_{1}, \ldots, Y_{m}\right\rangle$. Upon letting

$$
K\left(\left\langle Y_{1}, \ldots, Y_{m}\right\rangle\right)_{\sigma}=\tilde{\sigma}\left(K\left(\left\langle Y_{1}, \ldots, Y_{m}\right\rangle\right)\right)
$$

we get a field $K\left(\left\langle Y_{1}, \ldots, Y_{m}\right\rangle\right)_{\sigma}$. Clearly $\widetilde{\sigma}\left(\phi\left(K\left[\left[Y_{1}, \ldots, Y_{m}\right]\right]\right)\right)=\phi\left(K\left[\left[Y_{1}, \ldots, Y_{m}\right]\right]\right)$ and hence, interpreting $\subset$ to mean subring, we get

$$
\phi\left(K\left[\left[Y_{1}, \ldots, Y_{m}\right]\right]\right) \subset K\left(\left\langle Y_{1}, \ldots, Y_{m}\right\rangle\right)_{\sigma} .
$$

Therefore $\phi$ can be uniquely extended to a ring monomorphism

$$
\Phi_{\sigma}: K\left(\left(Y_{1}, \ldots, Y_{m}\right)\right) \rightarrow K\left(\left\langle Y_{1}, \ldots, Y_{m}\right\rangle\right)_{\sigma} .
$$

The paradox of Remark 1.5 is resolved by saying that for $\sigma \neq \tau$ in $S_{m}$ we need not have $\operatorname{im}\left(\Phi_{\sigma}\right)=\operatorname{im}\left(\Phi_{\tau}\right)$. This inspires

Question 4.1. Do we have $\bigcap_{\sigma \in S_{m}} K\left(\left\langle Y_{1}, \ldots, Y_{m}\right\rangle\right)_{\sigma}=\phi\left(K\left[\left[Y_{1}, \ldots, Y_{m}\right]\right]\right)$ ? 


\section{EXAMPLES OF NONMEROMORPHIC LEXOMORPHIC FUNCTIONS}

For $m>1$ we shall write down explicit examples of lexomorphic functions which are not meromorphic. Briefly speaking, we shall consider lots of lexomorphic functions whose supports are suitable disjoint infinite subsets of

$$
\Delta=\left\{(-n, n, 0, \ldots, 0): n \in \mathbb{N}_{+}\right\} \subset \mathbb{Z}^{m} .
$$

These will give you examples of infinitely many lexomorphic functions which are linearly independent over the meromorphic series field and, as we shall see in the next section, they can even be chosen to be algebraically independent over that field. Let us put

$$
C=\Phi\left(K\left[\left[Y_{1}, \ldots, Y_{m}\right]\right]\right)
$$

and

$$
E=\Phi\left(K\left(\left(Y_{1}, \ldots, Y_{m}\right)\right)\right) \subset K\left(\left\langle Y_{1}, \ldots, Y_{m}\right\rangle\right)=F .
$$

Now explicitly here are the:

Examples 5.1. Let $m>1$ and consider

$$
f=\sum_{1 \leq n<\infty} Y_{1}^{n^{2}} Y_{2}^{-n^{2}} \in F .
$$

We shall show that then $f \notin E$. Moreover, we shall show that $f$ gives rise to infinitely many members of $F$ which are linearly independent over $E$. Namely, partition the support of $f$ into infinitely many infinite disjoint subsets and let $f_{1}, f_{2}, \ldots$ be the members of $F$ having these supports. We shall show that then the elements $f_{1}, f_{2}, \ldots$ are linearly independent over $E$.

Definition 5.2. To establish the claims made in the above examples, we shall start by developing some sufficient conditions of linear independence. Let $p$ denote a positive integer.

Given any $\beta^{(j)}=\left(\beta_{1}^{(j)}, \ldots, \beta_{m}^{(j)}\right) \in \mathbb{Z}^{m}$ for $1 \leq j \leq p$, we put

$$
\beta^{(1)}+\cdots+\beta^{(p)}=\left(\beta_{1}^{(1)}+\cdots+\beta_{1}^{(p)}, \ldots, \beta_{m}^{(1)}+\cdots+\beta_{m}^{(p)}\right) \in \mathbb{Z}^{m} .
$$

Given any $D_{j} \subset \mathbb{Z}^{m}$ for $1 \leq j \leq p$, we put

$$
D_{1}+\cdots+D_{p}=\left\{\beta^{(1)}+\cdots+\beta^{(p)}: \beta^{(1)} \in D_{1}, \ldots, \beta^{(p)} \in D_{p}\right\} \subset \mathbb{Z}^{m} .
$$

Given any $\beta \in \mathbb{Z}^{m}$ and $D \subset \mathbb{Z}^{m}$ we put

$$
\beta+D=\{\beta+\gamma: \gamma \in D\} \subset \mathbb{Z}^{m} .
$$

Given any subset $D$ of $\mathbb{Z}^{m}$ we say that $D$ is uniclosed to mean that for every $\alpha \in \mathbb{N}^{m}$ there is a $\beta \in D$ such that $\beta+\alpha \notin \mathbb{N}^{m}$ and for all $\gamma \in D \backslash\{\beta\}$ we have $\beta+\alpha \notin \gamma+\mathbb{N}^{m}$.

Given any indexed family $D=\left(D_{w}\right)_{w \in W}$ of subsets $D_{w}$ of $\mathbb{Z}^{m}$ we say that the family is multiclosed to mean that: for all $w \neq w^{\prime}$ in $W$ we have $D_{w} \cap D_{w^{\prime}}=\emptyset$, and for every $(\alpha, w) \in \mathbb{N}^{m} \times W$ there is a $\beta \in D_{w}$ such that $\beta+\alpha \notin \mathbb{N}^{m}$ and for all $\gamma \in\left(\bigcup_{w^{\prime} \in W} D_{w^{\prime}}\right) \backslash\{\beta\}$ we have $\beta+\alpha \notin \gamma+\mathbb{N}^{m}$.

Given any overring $\bar{B}$ of a ring $\bar{C}$, an indexed family $\left(f_{w}\right)_{w \in W}$ of elements $f_{w}$ in $\bar{B}$ is said to be linearly overindependent over $\bar{C}$ to mean that: $\sum_{v \in V} h_{v} f_{v} \in \bar{C}$ with $h_{v} \in \bar{C}$ for all $v \in V=$ a finite subset of $W \Rightarrow h_{v}=0$ for all $v \in V$. Recall that the family $\left(f_{w}\right)_{w \in W}$ is said to be linearly independent over $\bar{C}$ to mean that: $\sum_{v \in V} h_{v} f_{v}=0$ with $h_{v} \in \bar{C}$ for all $v \in V=$ a finite subset $W \Rightarrow h_{v}=0$ for all 
$v \in V$. Consequently: linearly overindependent $\Rightarrow$ linearly independent. Also note that if $\bar{B}$ is a domain, then: linearly independent over $\bar{C} \Rightarrow$ linearly independent over the quotient field of $\bar{C}$.

We shall now prove the following theorem and then use it to establish the above explicit examples.

Theorem 5.3. As sufficient conditions of linear independence we have the following:

(5.3.1) If $f \in F$ is such that $\operatorname{Supp}(f)$ is uniclosed, then $f \notin E$.

(5.3.2) If $\left(f_{w}\right)_{w \in W}$ is a family in $F$ such that $\operatorname{Supp}\left(f_{w}\right)_{w \in W}$ is multiclosed, then the elements $\left(f_{w}\right)_{w \in W}$ are linearly overindependent over $C$.

Proof of (5.3.1). Let $f \in F$ be such that upon letting $D=\operatorname{Supp}(f)$ we have that $D$ is uniclosed. We want to prove that $f \notin E$. Assume the contrary. Then for some $0 \neq h \in C$ we have $h f \in C$. Since $0 \neq h \in C$, we can take $\alpha \in \mathbb{N}^{m}$ such that (i) $\alpha \in \operatorname{Supp}(h)$. Since $D$ is uniclosed, there is (ii) $\beta \in D$ such that (iii) $\beta+\alpha \notin \mathbb{N}^{m}$. Regarding $h f$ as a map $\mathbb{Z}^{m} \rightarrow K$ and calculating its value at $\beta+\alpha \in \mathbb{Z}^{m}$, by (i) and (ii) we get $(h f)(\beta+\alpha) \neq 0$. This is a contradiction because $h f \in C$ but $\beta+\alpha \notin \mathbb{N}^{m}$.

Proof of (5.3.2). Let $\left(f_{w}\right)_{w \in W}$ be a family of elements $f_{w} \in F$ such that, letting $D_{w}=\operatorname{Supp}\left(f_{w}\right)$ for all $w \in W$, the family $D=\left(D_{w}\right)_{w \in W}$ is multiclosed. We want to prove that the elements $\left(f_{w}\right)_{w \in W}$ are linearly overindependent over $C$.

Clearly it suffices to show that, given any finite subset $V$ of $W$ with $D_{w} \neq \emptyset$ for all $w \in V$ and given any $0 \neq h_{w} \in C$ for all $w \in V$ such that $\sum_{w \in V} h_{w} f_{w} \in C$, we must have $V=\emptyset$. Assume the contrary and fix $w \in V$. We can then find some $\alpha \in \operatorname{Supp}\left(h_{w}\right)$. Since $D$ is multiclosed, there is a $\beta \in D_{w}$ such that $\beta+\alpha \notin \mathbb{N}^{m}$ and for all $\gamma \in\left(\bigcup_{w^{\prime} \in W} D_{w^{\prime}}\right) \backslash\{\beta\}$ we have $\beta+\alpha \notin \gamma+\mathbb{N}^{m}$. It obviously follows that: $\left(1^{\bullet}\right)$ for all $\gamma \in\left(\bigcup_{w^{\prime} \in V} D_{w^{\prime}}\right) \backslash\{\beta\}$ we have $\beta+\alpha \notin \gamma+\mathbb{N}^{m}$. By definition of multiclosed we also have: $\left(2^{\bullet}\right) D_{w^{\prime}} \cap D_{w^{\prime \prime}}=\emptyset$ for all $w^{\prime} \neq w^{\prime \prime}$ in $V$. Regarding $h_{w^{\prime}} f_{w^{\prime}}$ as a map $\mathbb{Z}^{m} \rightarrow K$ and calculating its value at $\beta+\alpha \in \mathbb{Z}^{m}$, by $\left(1^{\bullet}\right)$ and $\left(2^{\bullet}\right)$ we see that: $\left(3^{\bullet}\right)\left(h_{w^{\prime}} f_{w^{\prime}}\right)(\beta+\alpha)=0$ for all $w^{\prime} \in V \backslash\{w\}$. Since $\alpha \in \operatorname{Supp}\left(h_{w}\right)$ and $\beta \in D_{w}$, by $\left(1^{\bullet}\right)$ we get: $\left(4^{\bullet}\right)\left(h_{w} f_{w}\right)(\beta+\alpha) \neq 0$. By $\left(3^{\bullet}\right)$ and $\left(4^{\bullet}\right)$ we conclude that: $\left(5^{\bullet}\right)\left(\sum_{w^{\prime} \in V} h_{w^{\prime}} f_{w^{\prime}}\right)(\beta+\alpha) \neq 0$. This is a contradiction because $\sum_{w^{\prime} \in V} h_{w^{\prime}} f_{w^{\prime}} \in C$ but $\beta+\alpha \notin \mathbb{N}^{m}$.

Lemma 5.4. Let $a: \mathbb{N}_{+} \rightarrow \mathbb{N}_{+}$be such that:

$$
\left\{\begin{array}{l}
\text { for all } n \in \mathbb{N}_{+} \text {we have } a(n)<a(n+1), \\
\text { and for every } \nu \in \mathbb{N}_{+} \text {there exists } \mu(\nu) \in \mathbb{N}_{+} \\
\text {such that } a(n)+\nu<a(n+1) \text { for all } n \geq \mu(\nu) .
\end{array}\right.
$$

Then, assuming $m>1$, and letting

$$
D=\left\{(a(n),-a(n), 0, \ldots, 0) \in \mathbb{Z}^{m}: n \in \mathbb{N}_{+}\right\}
$$

we have (5.4.1) to (5.4.3) stated below.

(5.4.1) Given any $\alpha \in \mathbb{N}^{m}$ and any infinite subset $\widehat{D}$ of $D$, there exists $\beta \in \widehat{D}$ such that $\beta+\alpha \notin \mathbb{N}^{m}$ and for all $\gamma \in D \backslash\{\beta\}$ we have $\beta+\alpha \notin \gamma+\mathbb{N}^{m}$.

(5.4.2) $D$ is uniclosed. 
(5.4.3) For all $i \in \mathbb{N}_{+}$let $D_{i}$ be an infinite subset of $D$ such that for all $i \neq j$ in $\mathbb{N}_{+}$we have $D_{i} \cap D_{j}=\emptyset$. Then $\left(D_{i}\right)_{i \in \mathbb{N}_{+}}$is a multiclosed indexed family of subsets of $\mathbb{Z}^{m}$.

(5.4.4) Without assuming $m>1$, if for some integer $k \geq 2$ we have $a(n)=k^{n}$ for all $n \in \mathbb{N}_{+}$, then a satisfies condition $(\dagger)$.

(5.4.5) Without assuming $m>1$, if for some integer $k \geq 2$ we have $a(n)=n^{k}$ for all $n \in \mathbb{N}_{+}$, then a satisfies condition $(\dagger)$.

Proof of (5.4.1). Now $\alpha=\left(\alpha_{1}, \ldots, \alpha_{m}\right)$ with $\alpha_{1}, \ldots, \alpha_{m}$ in $\mathbb{N}$. Letting $\nu=\alpha_{1}+$ $\alpha_{2}+1$ we get $\nu \in \mathbb{N}_{+}$. Since $\widehat{D}$ is assumed to be infinite, we can find $n \in \mathbb{N}_{+}$ such that $n>\mu(\nu)$ and upon letting $\beta=(a(n),-a(n), 0, \ldots, 0)$ we have $\beta \in \widehat{D}$. Now $\mu(\nu) \leq n-1 \in \mathbb{N}_{+}$and hence $a(n-1)+\nu<a(n)$ and therefore $\alpha_{2} \leq \nu \leq$ $a(n-1)+\nu<a(n)$. Hence $-a(n)+\alpha_{2}<0$ and therefore $\beta+\alpha \notin \mathbb{N}^{m}$. Given any $\gamma \in D \backslash\{\beta\}$, we have $\gamma=\left(a\left(n^{\prime}\right),-a\left(n^{\prime}\right), 0, \ldots, 0\right)$ with $n \neq n^{\prime} \in \mathbb{N}_{+}$. Clearly,

$$
n^{\prime}<n \Rightarrow \beta_{2}+\alpha_{2} \leq-a(n)+\nu<-a(n-1) \leq-a\left(n^{\prime}\right)=\gamma_{2} \Rightarrow \beta+\alpha \notin \gamma+\mathbb{N}^{m}
$$

and

$$
n^{\prime}>n \Rightarrow \beta_{1}+\alpha_{1} \leq a(n)+\nu<a(n+1) \leq \gamma_{1} \Rightarrow \beta+\alpha \notin \gamma+\mathbb{N}^{m} .
$$

Proof of (5.4.2). Take $D=\widehat{D}$ in (5.4.1).

Proof of (5.4.3). Given any $(\alpha, i) \in \mathbb{N}^{m} \times \mathbb{N}_{+}$, by taking $\widehat{D}=D_{i}$ in (5.4.1) we find $\beta \in D_{i}$ such that $\beta+\alpha \notin \mathbb{N}^{m}$ and for all $\gamma \in \mathbb{N}_{+} \backslash\{i\}$ we have $\beta+\alpha \notin \gamma+\mathbb{N}^{m}$. Therefore the family $\left(D_{i}\right)_{i \in \mathbb{N}_{+}}$is multiclosed.

Proof of (5.4.4) and (5.4.5). Take $\mu(\nu)=\nu$.

Examples 5.5. Let $a: \mathbb{N}_{+} \rightarrow \mathbb{N}_{+}$satisfy Lemma 5.4( $\dagger$. Let $m>1$ and consider

$$
f=\sum_{1 \leq n<\infty} Y_{1}^{a(n)} Y_{2}^{-a(n)} \in F .
$$

By (5.3.1) and (5.4.2) we see that $f \notin E$. Now let us partition the support of $f$ into infinitely many infinite disjoint subsets and let $f_{1}, f_{2}, \ldots$ be the members of $F$ having these supports; then by (5.3.2) and (5.4.3) we see that the elements $f_{1}, f_{2}, \ldots$ are linearly independent over $E$.

By (5.4.4) we see that, fixing any integer $k \geq 2$, we may take $a(n)=k^{n}$ for all $n \in \mathbb{N}_{+}$.

By (5.4.5) we see that, fixing any integer $k \geq 2$, we may take $a(n)=n^{k}$ for all $n \in \mathbb{N}_{+}$. The $k=2$ case of this corresponds to Examples 5.1.

Examples 5.6. To show that the converse of (5.3.1) is not true, let $a^{\prime}: N_{+} \rightarrow N_{+}$ be such that for all $n \in \mathbb{N}_{+}$we have $a^{\prime}(n)+2<a^{\prime}(n+1)$. Assuming $m>1$ let

$$
D^{\prime}=\left\{\beta_{n}^{\prime}: n \in \mathbb{N}_{+}\right\} \text {where } \beta_{n}^{\prime}=\left(a^{\prime}(n),-a^{\prime}(n), 0, \ldots, 0\right) \in \mathbb{Z}^{m}
$$

and

$$
D^{\prime \prime}=\left\{\beta_{n}^{\prime \prime}: n \in \mathbb{N}_{+}\right\} \text {where } \beta_{n}^{\prime \prime}=\left(a^{\prime}(n)+1,-a^{\prime}(n)-1,0, \ldots, 0\right) \in \mathbb{Z}^{m} .
$$

Clearly, $D^{\prime} \cap D^{\prime \prime}=\emptyset$. Taking $\alpha=(1,1,0, \ldots, 0) \in \mathbb{N}^{m}$ we see that for all $n \in \mathbb{N}_{+}$ we have

$$
\beta^{\prime}+\alpha=\beta_{n}^{\prime \prime}+(0,2,0, \ldots, 0) \quad \text { and } \quad \beta^{\prime \prime}+\alpha=\beta_{n}^{\prime}+(2,0,0, \ldots, 0)
$$

and hence $D^{\prime} \cup D^{\prime \prime}$ is NOT uniclosed. 
Let $a: \mathbb{N}_{+} \rightarrow \mathbb{N}_{+}$satisfy Lemma $5.4(\dagger)$ and take $a^{\prime}(n)=a(\mu(2)+n)$ for all $n \in \mathbb{N}_{+}$. Then clearly $a^{\prime}(n)+2<a^{\prime}(n+1)$ for all $n \in \mathbb{N}_{+}$and $a^{\prime}$ satisfies Lemma $5.4(\dagger)$. Therefore upon letting

$$
f^{\prime}=\sum_{1 \leq n<\infty} Y_{1}^{a^{\prime}(n)} Y_{2}^{-a^{\prime}(n)} \in F
$$

we have $\operatorname{Supp}\left(f^{\prime}\right)=D^{\prime}$; by (5.4.2) we see that $D^{\prime}$ is uniclosed and hence by (5.2.1) we conclude that $f^{\prime} \notin E$. Clearly $0 \neq 1+Y_{1} Y_{2}^{-1} \in E$ and hence upon letting $f^{\prime \prime}=\left(1+Y_{1} Y_{2}^{-1}\right) f^{\prime}$ we get $f^{\prime \prime} \in F \backslash E$. But obviously

$$
f^{\prime \prime}=f^{\prime}+Y_{1} Y_{2}^{-1} f^{\prime}=\sum_{1 \leq n<\infty} Y_{1}^{a^{\prime}(n)} Y_{2}^{-a^{\prime}(n)}+\sum_{1 \leq n<\infty} Y_{1}^{a^{\prime}(n)+1} Y_{2}^{-a^{\prime}(n)-1}
$$

and hence $\operatorname{Supp}\left(f^{\prime \prime}\right)=D^{\prime} \cup D^{\prime \prime}$ which shows that the converse of (5.3.1) is not true.

\section{EXAMPLES OF TRANSCENDENTAL LEXOMORPHIC FUNCTIONS}

The examples of the previous section can be modified to write down transcendental lexomorphic functions. Let us put

$$
C=\Phi\left(K\left[\left[Y_{1}, \ldots, Y_{m}\right]\right]\right)
$$

and

$$
E=\Phi\left(K\left(\left(Y_{1}, \ldots, Y_{m}\right)\right)\right) \subset K\left(\left\langle Y_{1}, \ldots, Y_{m}\right\rangle\right)=F .
$$

Now explicitly here are the:

Examples 6.1. Let $m>1$ and consider

$$
f=\sum_{1 \leq n<\infty} Y_{1}^{n^{n}} Y_{2}^{-n^{n}} \in F .
$$

We shall show that then $f$ is transcendental over $E$. Moreover, we shall show that $f$ gives rise to infinitely many members of $F$ which are algebraically independent over $E$. Namely, partition the support of $f$ into infinitely many infinite disjoint subsets and let $f_{1}, f_{2}, \ldots$ be the members of $F$ having these supports. We shall show that then the elements $f_{1}, f_{2}, \ldots$ are algebraically independent over $E$.

Definition 6.2. To establish the claims made in the above examples, we start by developing sufficient conditions of linear independence and algebraic independence.

For all $p$ and $j$ in $\mathbb{N}_{+}$we put

$$
N(p, j)=\left\{i \in \mathbb{N}^{p}: i_{1}+\cdots+i_{p}=j\right\} .
$$

Given any $n \in \mathbb{N}$ and $D \subset \mathbb{Z}^{m}$ we define $[n] D \subset \mathbb{Z}^{m}$ by putting

$$
[n] D= \begin{cases}D_{1}+\cdots+D_{n} \text { with } D_{1}=\cdots=D_{n}=D & \text { if } n>0, \\ \{(0, \ldots, 0)\} & \text { if } n=0 .\end{cases}
$$

Given any $i=\left(i_{1}, \ldots, i_{p}\right) \in \mathbb{N}^{p}$ with $p \in \mathbb{N}_{+}$and subsets $D_{1}, \ldots, D_{p}$ of $\mathbb{Z}^{m}$, we put

$$
[i]\left(D_{1}, \ldots, D_{p}\right)=\left[i_{1}\right] D_{1}+\cdots+\left[i_{p}\right] D_{p} .
$$

Given any set $W$ and any $p \in \mathbb{N}_{+}$, by $W^{(p)}$ we denote the set of all sequences $v=\left(v_{1}, \ldots, v_{p}\right)$ of distinct elements $v_{1}, \ldots, v_{p}$ in $W$. For any such $p$ and $v$, and any indexed family $\left(D_{w}\right)_{w \in W}$ of subsets $D_{w}$ of $\mathbb{Z}^{m}$ and $i \in \mathbb{N}^{p}$ we put

$$
D_{i}^{(v)}=[i]\left(D_{v_{1}}, \ldots, D_{v_{p}}\right) \text {. }
$$


By a boxing of a set $U$ we mean a family $\left(U_{j}\right)_{j \in \mathbb{N}_{+}}$of subsets $U_{j}$ of $U$ such that $U=\bigcup_{j \in \mathbb{N}_{+}} U_{j}$ and $U_{j} \cap U_{j^{\prime}}=\emptyset$ for all $j \neq j^{\prime}$ in $\mathbb{N}_{+}$.

To sharpen Theorem 5.3, we first sharpen the concept of multiclosed thus. By a polyfamily we mean an indexed family $D=\left(\widehat{D}_{i}, D_{i}\right)_{i \in U}$ of subsets $\widehat{D}_{i}$ and $D_{i}$ of $\mathbb{Z}^{m}$ together with a boxing $\left(U_{j}\right)_{j \in \mathbb{N}_{+}}$of $U$. We say that such a polyfamily $D$ is polyclosed to mean that: for all $i \in U$ we have $\widehat{D}_{i} \subset D_{i}$, and for every $(\alpha, j, i) \in \mathbb{N}^{m} \times \mathbb{N}_{+} \times U_{j}$ there is a $\beta \in \widehat{D}_{i}$ such that $\beta+\alpha \notin \mathbb{N}^{m}$ and for all $\gamma \in\left(\bigcup_{i^{\prime} \in\left(U_{1} \cup \ldots \cup U_{j}\right) \backslash\{i\}} D_{i^{\prime}}\right) \cup\left(D_{i} \backslash\{\beta\}\right)$ we have $\beta+\alpha \notin \gamma+\mathbb{N}^{m}$.

Sharpening Theorem 5.3 we get

Theorem 6.3. As sufficient conditions of linear independence and algebraic independence we have the following:

(6.3.1) Let $\left(f_{i}\right)_{i \in U}$ be a family of elements $f_{i}$ in $F$. Assume that there exists a polyclosed polyfamily $D$ as above such that for all $i \in U$ we have $\operatorname{Supp}\left(f_{i}\right)=\widehat{D}_{i}$. Then the elements $\left(f_{i}\right)_{i \in U}$ are linearly overindependent over $C$.

(6.3.2) Given any $f \in F$, let $\left(U_{j}\right)_{j \in \mathbb{N}_{+}}$be the boxing of $U=\mathbb{N}_{+}$given by putting $U_{j}=\{j\}$ for all $j \in \mathbb{N}_{+}$, consider the polyfamily $D^{*}=\left(\widehat{D}_{n}^{*}, D_{n}^{*}\right)_{n \in U}$ obtained by putting $\widehat{D}_{n}^{*}=\operatorname{Supp}\left(f^{n}\right)$ and $D_{n}^{*}=[n] \operatorname{Supp}(f)$ for all $n \in \mathbb{N}_{+}$, and assume that the polyfamily $D^{*}$ is polyclosed. Then $f$ is transcendental over $E$.

$N_{\text {ote }}$. In the proof we shall tacitly use the following facts: Let $\bar{C}$ be any domain with quotient field $\bar{E}$, let $\bar{F}$ be any overfield of $\bar{E}$, and let $f$ be any element of $\bar{F}$. Then $f$ is transcendental over $E \Leftrightarrow$ the elements $\left(f^{n}\right)_{n \in \mathbb{N}_{+}}$are linearly overindependent over $\bar{C}$. Moreover, in (6.3.2) we have $\widehat{D}_{n}^{*} \subset D_{n}^{*}$ for all $n \in \mathbb{N}_{+}$.

(6.3.3) Given any indexed family $\left(f_{w}\right)_{w \in W}$ of elements $f_{w}$ in $F$, let $D=\left(D_{w}\right)_{w \in W}$ be the family of subsets of $\mathbb{Z}^{m}$ obtained by putting $D_{w}=\operatorname{Supp}\left(f_{w}\right)$ for all $w \in W$. For any $p \in \mathbb{N}_{+}$and $v \in W^{(p)}$, let $\left(U_{j}\right)_{j \in \mathbb{N}_{+}}$be the boxing of $U=\mathbb{N}^{p} \backslash\{(0, \ldots, 0)\}$ given by putting $U_{j}=N(p, j)$ for all $j \in \mathbb{N}_{+}$, and consider the polyfamily $D^{(v)}=$ $\left(\widehat{D}_{i}^{(v)}, D_{i}^{(v)}\right)_{i \in U}$ obtained by putting $\widehat{D}_{i}^{(v)}=\operatorname{Supp}\left(f_{v_{1}}^{i_{1}} \ldots f_{v_{p}}^{i_{p}}\right)$ for all $i \in U$. Assume that for all $p \in \mathbb{N}_{+}$and $v \in W^{(p)}$, the polyfamily $D^{(v)}$ is polyclosed. Then the elements $\left(f_{w}\right)_{w \in W}$ are algebraically independent over $E$.

$N o t e^{* *}$. In the proof we shall tacitly use the following facts: Let $\bar{C}$ be any domain with quotient field $\bar{E}$, let $\bar{F}$ be any overfield of $\bar{E}$, and let $\left(f_{w}\right)_{w \in W}$ be any indexed family of elements $f_{w}$ in $\bar{F}$. Then the elements $\left(f_{w}\right)_{w \in W}$ are algebraically independent over $\bar{E} \Leftrightarrow$ for every $j \in \mathbb{N}_{+}$and every finite sequence $v_{1}, \ldots, v_{p}$ of distinct elements in $W$, the monomials $\left(f_{v_{1}}^{i_{1}} \ldots f_{v_{p}}^{i_{p}}\right)_{1 \leq i_{1}+\cdots+i_{p} \leq j}$ are linearly overindependent over $\bar{C}$. Moreover, in (6.3.3) we have $\widehat{D}_{i}^{(v)} \subset D_{i}^{(v)}$ for all $v \in W^{(p)}$ and $i \in \mathbb{N}^{p}$ with $p \in \mathbb{N}_{+}$.

Proof of (6.3.1). Clearly it suffices to show that, given any finite subset $V$ of $U$ with $\widehat{D}_{i} \neq \emptyset$ for all $i \in V$ and given any $0 \neq h_{i} \in C$ for all $i \in V$ such that $\sum_{i \in V} h_{i} f_{i} \in C$, we must have $V=\emptyset$. Assuming the contrary, let $j$ be the largest positive integer such that $V \cap U_{j} \neq \emptyset$ and fix $i \in V \cap U_{j}$. We can then find some $\alpha \in \operatorname{Supp}\left(h_{i}\right)$. Since $D$ is polyclosed, there is a $\beta \in \widehat{D}_{i}$ such that $\beta+\alpha \notin \mathbb{N}^{m}$ and: $\left(1^{\bullet}\right)$ for all $\gamma \in\left(\bigcup_{i^{\prime} \in\left(U_{1} \cup \cdots \cup U_{j}\right) \backslash\{i\}} D_{i^{\prime}}\right) \cup\left(D_{i} \backslash\{\beta\}\right)$ we have $\beta+\alpha \notin \gamma+\mathbb{N}^{m}$. Regarding $h_{i^{\prime}} f_{i^{\prime}}$ as a map $\mathbb{Z}^{m} \rightarrow K$ and calculating its value at $\beta+\alpha \in \mathbb{Z}^{m}$, by $\left(1^{\bullet}\right)$ we see that: $\left(2^{\bullet}\right)\left(h_{i^{\prime}} f_{i^{\prime}}\right)(\beta+\alpha)=0$ for all $i^{\prime} \in V \backslash\{i\}$. Since $\alpha \in \operatorname{Supp}\left(h_{i}\right)$ and 
$\beta \in \widehat{D}_{i}$, by $\left(1^{\bullet}\right)$ we get: $\left(3^{\bullet}\right)\left(h_{i} f_{i}\right)(\beta+\alpha) \neq 0$. By $\left(2^{\bullet}\right)$ and $\left(3^{\bullet}\right)$ we conclude that: $\left(4^{\bullet}\right)\left(\sum_{i^{\prime} \in V} h_{i^{\prime}} f_{i^{\prime}}\right)(\beta+\alpha) \neq 0$. This is a contradiction because $\sum_{i^{\prime} \in V} h_{i^{\prime}} f_{i^{\prime}} \in C$ but $\beta+\alpha \notin \mathbb{N}^{m}$.

Proof of (6.3.2). In view of Note*, it suffices to show that the elements $\left(f^{n}\right)_{n \in N_{+}}$ are linearly overindependent over $C$. This follows by taking $D^{*}$ for $D$ in (6.3.1).

Proof of (6.3.3). In view of Note**, it suffices to show that for every $j \in \mathbb{N}_{+}$ and every finite sequence $v_{1}, \ldots, v_{p}$ of distinct elements in $W$, the monomials $\left(f_{v_{1}}^{i_{1}} \ldots f_{v_{p}}^{i_{p}}\right)_{1 \leq i_{1}+\cdots+i_{p} \leq j}$ are linearly overindependent over $C$. This follows by taking $D^{(v)}$ for $D$ in $(6.3 .1)$.

Lemma 6.4. Let $b: \mathbb{N}_{+} \rightarrow \mathbb{N}_{+}$be such that

$$
\left\{\begin{array}{l}
\text { for all } n \in \mathbb{N}_{+} \text {we have } b(n)<b(n+1), \\
\text { and } b(n+1) / b(n) \text { tends to } \infty \text { as } n \text { tends to } \infty .
\end{array}\right.
$$

Then, given any $\nu \in \mathbb{N}_{+}$and $\nu^{\prime} \in \mathbb{N}_{+}$, there exists $\mu\left(\nu, \nu^{\prime}\right) \in \mathbb{N}_{+}$such that for all $n \geq \mu\left(\nu, \nu^{\prime}\right)$ we have $b(n+1) / b(n)>\nu\left(\nu^{\prime}+1\right)$. Moreover, for every such $\mu\left(\nu, \nu^{\prime}\right)$ we have that: if $\mu\left(\nu, \nu^{\prime}\right)<n_{1} \leq n_{2} \leq \cdots \leq n_{r}$ and $1 \leq m_{1} \leq m_{2} \leq \cdots \leq m_{s}$ are any integers with $1 \leq s \leq r \leq \nu$ and $\left(n_{1}, \ldots, n_{r}\right) \neq\left(m_{1}, \ldots, m_{s}\right)$, then $\left|n^{\prime}-m^{\prime}\right|>\nu^{\prime}$ where $n^{\prime}=b\left(n_{1}\right)+\cdots+b\left(n_{r}\right)$ and $m^{\prime}=b\left(m_{1}\right)+\cdots+b\left(m_{s}\right)$.

(Here $\|$ denotes norm, and $\left(n_{1}, \ldots, n_{r}\right)=\left(m_{1}, \ldots, m_{s}\right)$ means $r=s$ and $n_{i}=m_{i}$ for $1 \leq i \leq r$.)

Proof. We are assuming that $b(n+1) / b(n) \rightarrow \infty$ as $n \rightarrow \infty$, and

(1) $b(n)<b(n+1)$ for all $n \in \mathbb{N}_{+}$.

These assumptions tell us that, given any $\nu \in \mathbb{N}_{+}$and $\nu^{\prime} \in \mathbb{N}_{+}$, there exists $\mu\left(\nu, \nu^{\prime}\right) \in \mathbb{N}_{+}$such that

(2) for all $n \in \mathbb{N}_{+}$with $n \geq \mu\left(\nu, \nu^{\prime}\right)$ we have $b(n+1)>\nu\left(\nu^{\prime}+1\right) b(n)$.

Now given any integers

(3) $\mu\left(\nu, \nu^{\prime}\right)<n_{1} \leq n_{2} \leq \cdots \leq n_{r}$, and

(4) $1 \leq m_{1} \leq m_{2} \leq \cdots \leq m_{s}$ with $1 \leq s \leq r \leq \nu$, we want to show that

$$
\left(n_{1}, \ldots, n_{r}\right) \neq\left(m_{1}, \ldots, m_{s}\right) \Rightarrow\left|n^{\prime}-m^{\prime}\right|>\nu^{\prime} .
$$

We shall prove $(\bullet)$ by induction on $r$. By (1) to (3) we see that

(5) for all $n \in \mathbb{N}_{+}$with $n>n_{r}$ we have $b(n)>\nu\left(\nu^{\prime}+1\right) b\left(n_{r}\right)$, and

(6) for all $n \in \mathbb{N}_{+}$with $n<n_{r}$ we have $\nu\left(\nu^{\prime}+1\right) b(n)<b\left(n_{r}\right)$.

In view of (1) to (4), respectively by (5) and (6) we see that

(7) $m_{s}>n_{r} \Rightarrow m^{\prime} \geq b\left(m_{s}\right)>\nu\left(\nu^{\prime}+1\right) b\left(n_{r}\right) \geq\left(\nu^{\prime}+1\right) n^{\prime} \geq n^{\prime}+\nu^{\prime}$, and

(8) $m_{s}<n_{r} \Rightarrow m^{\prime}+\nu^{\prime} \leq\left(\nu^{\prime}+1\right) m^{\prime} \leq \nu\left(\nu^{\prime}+1\right) b\left(m_{s}\right)<b\left(n_{r}\right) \leq n^{\prime}$.

Now if $\left(n_{1}, \ldots, n_{r}\right) \neq\left(m_{1}, \ldots, m_{s}\right)$ and $r=1=s$, then clearly $m_{s} \neq n_{r}$; consequently, by $(7)$ and $(8)$ we see that

(9) $\left(n_{1}, \ldots, n_{r}\right) \neq\left(m_{1}, \ldots, m_{s}\right)$ with $r=1=s \Rightarrow\left|n^{\prime}-m^{\prime}\right|>\nu^{\prime}$.

By (2) we see that if $r>1$, then $b\left(n_{r-1}\right)>\nu^{\prime}$, and hence

(10) if $r>1=s$ with $m_{s}=n_{r}$, then $n^{\prime}-m^{\prime}=b\left(n_{1}\right)+\cdots+b\left(n_{r-1}\right)>\nu^{\prime}$. 
Obviously,

$$
\left\{\begin{array}{l}
\text { if } r>1<s \text { with } m_{s}=n_{r}, \text { then: } \\
\left(n_{1}, \ldots, n_{r}\right) \neq\left(m_{1}, \ldots, m_{s}\right) \Leftrightarrow\left(n_{1}, \ldots, n_{r-1}\right) \neq\left(m_{1}, \ldots, m_{s-1}\right) \\
\text { and } n^{\prime}-m^{\prime}=b\left(n_{1}\right)+\cdots+b\left(n_{r-1}\right)-b\left(m_{1}\right)-\cdots-b\left(m_{s-1}\right) .
\end{array}\right.
$$

In case of $r=1,(\bullet)$ follows from (9).

In case of $r>1$, by (7), (8), (10), (11) we see that: $(\bullet)$ for $r-1 \Rightarrow(\bullet)$ for $r$.

Lemma 6.5. Let $b: \mathbb{N}_{+} \rightarrow \mathbb{N}_{+}$satisfy Lemma $6.4(\sharp)$. In case of $m>1$, for every $n \in \mathbb{N}_{+}$we put $\delta(n)=(n,-n, 0, \ldots, 0) \in \mathbb{Z}^{m}$. Then, assuming $m>1$, and letting $\widetilde{D}=\left\{\delta(n): n \in \mathbb{N}_{+}\right\} \subset \mathbb{Z}^{m}$, we have (6.5.1) and (6.5.2) stated below.

(6.5.1) Let $f \in F$ be such that $\operatorname{Supp}(f)=\widetilde{D}$. Then $f$ is transcendental over $E$.

(6.5.2) Let $W=\mathbb{N}_{+}$. For all $w \in W$ let $D_{i}$ be an infinite subset of $\widetilde{D}$ such that for all $w \neq w^{\prime}$ in $W$ we have $D_{w} \cap D_{w^{\prime}}=\emptyset$. For every $w \in W$ let $f_{w} \in F$ be such that $\operatorname{Supp}\left(f_{w}\right)=D_{w}$. Then the elements $f_{1}, f_{2}, \ldots$ are algebraically independent over $E$.

(6.5.3) Without assuming $m>1$, if $b(n)=n$ ! for all $n \in \mathbb{N}_{+}$, then $b$ satisfies Lemma $6.4(\sharp)$.

(6.5.4) Without assuming $m>1$, if $b(n)=n^{n}$ for all $n \in \mathbb{N}_{+}$, then $b$ satisfies Lemma $6.4(\sharp)$.

Proof of (6.5.1). Let $\left(U_{j}\right)_{j \in \mathbb{N}_{+}}$be the boxing of $U=\mathbb{N}_{+}$given by putting $U_{j}=\{j\}$ for all $j \in \mathbb{N}_{+}$, and consider the polyfamily $D^{*}=\left(\widehat{D}_{i}^{*}, D_{i}^{*}\right)_{i \in U}$ obtained by putting $\widehat{D}_{i}^{*}=\operatorname{Supp}\left(f^{i}\right)$ and $D_{i}^{*}=[i] \operatorname{Supp}(f)$ for all $i \in \mathbb{N}_{+}$. To prove $f$ is transcendental over $E$, in view of (6.3.2) it suffices to show that $D^{*}$ is polyclosed. By Note* we have $\widehat{D}_{i}^{*} \subset D_{i}^{*}$ for all $i \in \mathbb{N}_{+}$. It only remains to show that given any $(\alpha, i) \in \mathbb{N}^{m} \times \mathbb{N}_{+}$ there is a $\beta \in \operatorname{Supp}\left(f^{i}\right)$ such that $\beta+\alpha \notin \mathbb{N}^{m}$ and for all $\gamma \in D_{1}^{*} \cup \cdots \cup D_{i-1}^{*} \cup$ $\left(D_{i}^{*} \backslash\{\beta\}\right)$ we have $\beta+\alpha \notin \gamma+\mathbb{N}^{m}$. Taking

$$
\nu=\nu^{\prime}=\alpha_{1}+\alpha_{2}+i
$$

in Lemma 6.4 we find $\mu(\nu, \nu) \in \mathbb{N}_{+}$such that

$$
\text { for all } n \geq \mu(\nu, \nu) \text { we have } b(n+1) / b(n)>\nu(\nu+1) \text {. }
$$

By Lemma 6.4 we know that

$$
\left\{\begin{array}{l}
\text { if } \mu(\nu, \nu)<n_{1} \leq n_{2} \leq \cdots \leq n_{r} \text { and } 1 \leq m_{1} \leq m_{2} \leq \cdots \leq m_{s} \\
\text { are any integers with } 1 \leq s \leq r \leq \nu \\
\text { and }\left(n_{1}, \ldots, n_{r}\right) \neq\left(m_{1}, \ldots, m_{s}\right), \text { then }\left|n^{\prime}-m^{\prime}\right|>\nu \\
\text { where } n^{\prime}=b\left(n_{1}\right)+\cdots+b\left(n_{r}\right) \text { and } m^{\prime}=b\left(m_{1}\right)+\cdots+b\left(m_{s}\right) .
\end{array}\right.
$$

Fixing any integer

$$
n>\mu(\nu, \nu)
$$

and letting

$$
n_{1}=\cdots=n_{i}=n \quad \text { and } \quad n^{\prime}=i b(n)
$$


by $(\ddagger)$ we see that

$$
\left\{\begin{array}{l}
\text { if } 1 \leq m_{1} \leq \cdots \leq m_{s} \text { are any integers with } 1 \leq s \leq i \\
\text { and }\left(n_{1}, \ldots, n_{i}\right) \neq\left(m_{1}, \ldots, m_{s}\right) \text {, then }\left|n^{\prime}-m^{\prime}\right|>\nu \\
\text { where } m^{\prime}=b\left(m_{1}\right)+\cdots+b\left(m_{s}\right) .
\end{array}\right.
$$

Since $\operatorname{Supp}(f)=\widetilde{D}$, upon letting $\beta=\delta\left(n^{\prime}\right)$, by (4) and the $s=i$ case of (5) we conclude that $\beta \in \operatorname{Supp}\left(f^{i}\right)$. Mimicking the proof of (5.4.1) we shall show that $\beta+\alpha \notin \mathbb{N}^{m}$ and for all $\gamma \in D_{1}^{*} \cup \cdots \cup D_{i-1}^{*} \cup\left(D_{i}^{*} \backslash\{\beta\}\right)$ we have $\beta+\alpha \notin \gamma+\mathbb{N}^{m}$. This will complete the proof.

By (2) and (3) we have $n^{\prime}>\nu$, and by (1) we have $\nu>\alpha_{2}$; consequently, $n^{\prime}>\alpha_{2}$; but by definition $\beta_{2}=-n^{\prime}$ and hence $\beta_{2}+\alpha_{2}<0$ and therefore $\beta+\alpha \notin \mathbb{N}^{m}$. Given any $\gamma \in D_{1}^{*} \cup \cdots \cup D_{i-1}^{*} \cup\left(D_{i}^{*} \backslash\{\beta\}\right)$, we may assume that in the situation of (5) we have $\gamma=\delta\left(m^{\prime}\right)$; now it suffices to note that

$$
m^{\prime}<n^{\prime} \Rightarrow \beta_{2}+\alpha_{2}=-n^{\prime}+\alpha_{2} \leq-n^{\prime}+\nu<-m^{\prime}=\gamma_{2} \Rightarrow \beta+\alpha \notin \gamma+\mathbb{N}^{m}
$$

and

$$
m^{\prime}>n^{\prime} \Rightarrow \beta_{1}+\alpha_{1}=n^{\prime}+\alpha_{1} \leq n^{\prime}+\nu<m^{\prime}=\gamma_{1} \Rightarrow \beta+\alpha \notin \gamma+\mathbb{N}^{m} .
$$

Proof of (6.5.2). For any $p \in \mathbb{N}_{+}$and any sequence $v=\left(v_{1}, \ldots, v_{p}\right)$ of distinct positive integers, let $\left(U_{j}\right)_{j \in \mathbb{N}_{+}}$be the boxing of $U=\mathbb{N}^{p} \backslash\{(0, \ldots, 0)\}$ given by putting $U_{j}=N(p, j)$ for all $j \in \mathbb{N}_{+}$, and consider the polyfamily $D^{(v)}=\left(\widehat{D}_{i}^{(v)}, D_{i}^{(v)}\right)_{i \in U}$ obtained by putting $\widehat{D}_{i}^{(v)}=\operatorname{Supp}\left(f_{v_{1}}^{i_{1}} \ldots f_{v_{p}}^{i_{p}}\right)$ for all $i \in U$. To prove that the elements $f_{1}, f_{2}, \ldots$ are algebraically independent over $E$, in view of (6.3.3) it suffices to show that $D^{(v)}$ is polyclosed. By Note** we have $\widehat{D}_{i}^{(v)} \subset D_{i}^{(v)}$ for all $i \in U$. It only remains to show that given any $(\alpha, j, i) \in \mathbb{N}^{m} \times \mathbb{N}_{+} \times N(p, j)$ there is a $\beta \in \widehat{D}_{i}^{(v)}$ such that $\beta+\alpha \notin \mathbb{N}^{m}$ and for all $\gamma \in\left(\bigcup_{i^{\prime} \in\left(U_{1} \cup \cdots \cup U_{j}\right) \backslash\{i\}} D_{i^{\prime}}\right) \cup\left(D_{i} \backslash\{\beta\}\right)$ we have $\beta+\alpha \notin \gamma+\mathbb{N}^{m}$. Taking

$$
\nu=\nu^{\prime}=\alpha_{1}+\alpha_{2}+j
$$

in Lemma 6.4 we find $\mu(\nu, \nu) \in \mathbb{N}_{+}$such that

$$
\text { for all } n \geq \mu(\nu, \nu) \text { we have } b(n+1) / b(n)>\nu(\nu+1) \text {. }
$$

By Lemma 6.4 we know that

$$
\left\{\begin{array}{l}
\text { if } \mu(\nu, \nu)<n_{1} \leq n_{2} \leq \cdots \leq n_{r} \text { and } 1 \leq m_{1} \leq m_{2} \leq \cdots \leq m_{s} \\
\text { are any integers with } 1 \leq s \leq r \leq \nu \\
\text { and }\left(n_{1}, \ldots, n_{r}\right) \neq\left(m_{1}, \ldots, m_{s}\right) \text {, then }\left|n^{\prime}-m^{\prime}\right|>\nu \\
\text { where } n^{\prime}=b\left(n_{1}\right)+\cdots+b\left(n_{r}\right) \text { and } m^{\prime}=b\left(m_{1}\right)+\cdots+b\left(m_{s}\right) .
\end{array}\right.
$$

Clearly, we can find integers $n_{1}^{\prime}, \ldots, n_{p}^{\prime}$ such that

$$
\mu(\nu, \nu)<n_{1}^{\prime}<\cdots<n_{p}^{\prime} \text { with } \delta\left(n_{q}^{\prime}\right) \in D_{v_{q}} \text { for } 1 \leq q \leq p
$$

and now letting

(4)

$$
n_{l}=n_{q}^{\prime} \text { for } 1 \leq q \leq p \quad \text { and } \quad \sum_{1 \leq t \leq q-1} i_{t}<l \leq \sum_{1 \leq t \leq q} i_{t} \text { with } n^{\prime}=\sum_{1 \leq q \leq p} i_{q} b\left(n_{q}^{\prime}\right)
$$


by $(\ddagger)$ we see that

$$
\left\{\begin{array}{l}
\text { if } 1 \leq m_{1} \leq \cdots \leq m_{s} \text { are any integers with } 1 \leq s \leq j \\
\text { and }\left(n_{1}, \ldots, n_{j}\right) \neq\left(m_{1}, \ldots, m_{s}\right), \text { then }\left|n^{\prime}-m^{\prime}\right|>\nu \\
\text { where } m^{\prime}=b\left(m_{1}\right)+\cdots+b\left(m_{s}\right) .
\end{array}\right.
$$

Since $\operatorname{Supp}\left(f_{v_{q}}\right)=D_{v_{q}}$ for $1 \leq q \leq p$, upon letting $\beta=\delta\left(n^{\prime}\right)$, by (4) and the $s=j$ case of (5) we conclude that $\beta \in \widehat{D}_{i}^{(v)}$. Mimicking the proof of (5.4.1) we shall show that $\beta+\alpha \notin \mathbb{N}^{m}$ and for all $\gamma \in\left(\bigcup_{i^{\prime} \in\left(U_{1} \cup \ldots \cup U_{j}\right) \backslash\{i\}} D_{i^{\prime}}\right) \cup\left(D_{i} \backslash\{\beta\}\right)$ we have $\beta+\alpha \notin \gamma+\mathbb{N}^{m}$. This will complete the proof.

By (2) to (4) we have $n^{\prime}>\nu$, and by (1) we have $\nu>\alpha_{2}$; consequently, $n^{\prime}>\alpha_{2}$; but by definition $\beta_{2}=-n^{\prime}$. Hence $\beta_{2}+\alpha_{2}<0$ and therefore $\beta+\alpha \notin \mathbb{N}^{m}$. Given any $\gamma \in\left(\bigcup_{i^{\prime} \in\left(U_{1} \cup \ldots \cup U_{j}\right) \backslash\{i\}} D_{i^{\prime}}\right) \cup\left(D_{i} \backslash\{\beta\}\right)$ we may assume that in the situation of (5) we have $\gamma=\delta\left(m^{\prime}\right)$; now it suffices to note that

$$
m^{\prime}<n^{\prime} \Rightarrow \beta_{2}+\alpha_{2}=-n^{\prime}+\alpha_{2} \leq-n^{\prime}+\nu<-m^{\prime}=\gamma_{2} \Rightarrow \beta+\alpha \notin \gamma+\mathbb{N}^{m}
$$

and

$$
m^{\prime}>n^{\prime} \Rightarrow \beta_{1}+\alpha_{1}=n^{\prime}+\alpha_{1} \leq n^{\prime}+\nu<m^{\prime}=\gamma_{1} \Rightarrow \beta+\alpha \notin \gamma+\mathbb{N}^{m} .
$$

Proof of (6.5.3) and (6.5.4). Obvious.

Examples 6.6. Let $b: \mathbb{N}_{+} \rightarrow \mathbb{N}_{+}$satisfy Lemma $6.5(\sharp)$. Let $m>1$ and consider

$$
f=\sum_{1 \leq n<\infty} Y_{1}^{b(n)} Y_{2}^{-b(n)} \in F .
$$

By (6.5.1) we see that $f$ is transcendental over $E$. Now partition the support of $f$ into infinitely many infinite disjoint subsets and let $f_{1}, f_{2}, \ldots$ be the members of $F$ having these supports; then by (6.5.2) we see that the elements $f_{1}, f_{2}, \ldots$ are algebraically independent over $E$.

By (6.5.3) we see that we may take $b(n)=n$ ! for all $n \in \mathbb{N}_{+}$.

By (6.5.4) we see that we may take $b(n)=n^{n}$ for all $n \in \mathbb{N}_{+}$; this corresponds to Examples 6.1.

Question 6.7. In the situation of Examples 6.6 we may ask whether $E$ is relatively algebraically closed in $F$. As a partial answer let $f=\sum_{1<n<\infty} Y_{1}^{2^{n}} Y_{2}^{-2^{n}}$ and assume $K$ is of characteristic 2. By Examples 5.5 we see that $f \in F \backslash E$, but clearly $f^{2}+f+Y_{1}^{2} Y_{2}^{-2}=0$ and hence $[E(f): E]=2$.

\section{REFERENCES}

[Ab1] Shreeram S. Abhyankar, Algebraic geometry for scientists and engineers, Mathematical Surveys and Monographs, vol. 35, American Mathematical Society, Providence, RI, 1990. MR.1075991 (92a:14001)

[Ab2] S. S. Abhyankar, Lectures on Algebra I, World Scientific, 2006.

Department of Mathematics, Purdue University, West Lafayette, Indiana 47907

E-mail address: ram@cs.purdue.edu

Department of Mathematics, Purdue University, West Lafayette, Indiana 47907

E-mail address: nfireman@math.purdue.edu

Department of Mathematics, Purdue University, West Lafayette, Indiana 47907

E-mail address: nmoses@math.purdue.edu 